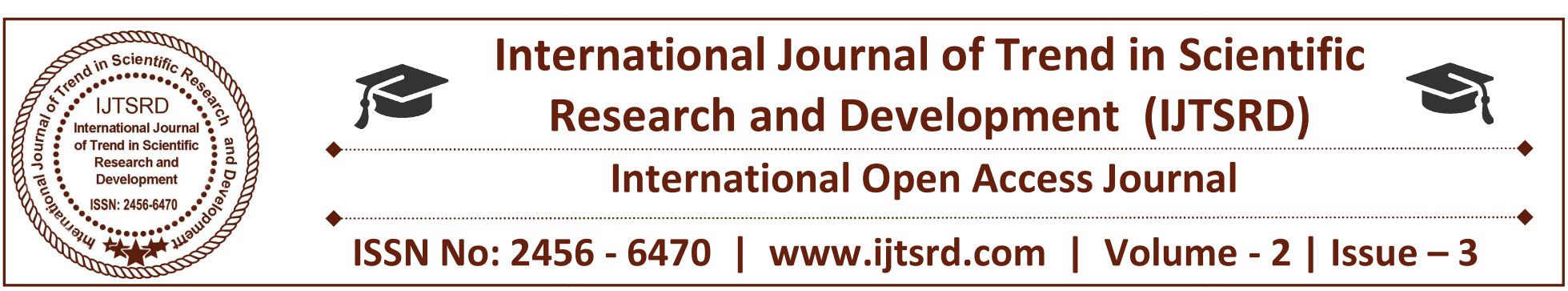

\title{
Employee Data Mining Based on Text and Image Processing
}

\author{
Prof. Vijay More, Ms. Ankita Shetty, Ms. Aishwarya Mapara, Mr. Rahul Ghuge, Mr. Rohit Sharma \\ Department of Information Technology, AISSMS Institute of Information Technology \\ Pune, Maharashtra, India
}

\begin{abstract}
Employees of any company need to know whether their employees are happy or sad or they have any problem in their working environment. There should be some mechanism to handle this information about the employee. Employee chatting messages could be analyzed using sentiment analysis and employee mood detection is retrieved based on text analysis. Also, Employee facial expressions can be detected using Image Processing on employee images taken through Web Camera while an employee is chatting with colleagues. Using Image Processing, Emotion of employee such as Happy, Angry, Sad or Normal is detected. Employee analysis report is shown to company management to find whether the employee is satisfied with company or employee is facing some problem in the working environment.
\end{abstract}

\section{INTRODUCTION}

Social Networking Portal for Employees of any company is being used extensively nowadays in corporate world. Companies need to know whether their employees are happy or sad or they have any problem in their working environment.

There should be some mechanism to handle this information about employee. Employee chatting messages could be analyzed using sentiment analysis and employee mood detection is retrieved based on text analysis. Also, Employee facial expressions can be detected using Image Processing on employee images taken through Web Camera while employee is chatting with colleagues. Using Image Processing, Emotion of employee such as Happy, Angry, Sad or Normal is detected. Employee analysis report is shown to company management to find whether employee is satisfied with company or employee is facing some problem in working environment. Image processing is a method to convert an image into digital form and perform some operations on it, in order to get an enhanced image or to extract some useful information from it. In this technique we analyze an employee's expression, when they are talking with any person.

\section{Literature Survey}

\section{A. Employee Monitoring and HR Mangement Using RFID.}

Author: S. Srinivasan, Dr. H. Ranganathan, R. Srivel. This paper states that using RFID person or object are identified over radio frequencies. By using RFID there is no manually tags or label scanned for extracting information tags are used. Tags are unique. It is tracking application it is used inside the campus or offices.

\section{B. New Distances Combination for Facial Expression Recognition from image sequence.}

Author: Fatima Zahra Salmam, Abdellah Madani, and Mohamed Kissi. This paper illustrate that recognize from image sequence take the first and last frame of the facial expression it represent emotion and neutral state. These methods calculate the distances there are six distances of the eleven points. These points are calculated by support descent method ( SDM).By calculating these points we get the emotion of the face whether the human is happy or sad. 


\section{Image Data Mining From Finanical Document Based On Wavelet Feature}

Author: O.El Badawy, M.R. El-Sakka, K.Hassanein, M. S.Kamel. We present a framework for clustering and classifying cheque images according to their payee-line content. The features used in the clustering and classification processes are extracted from the wavelet domain by means of threesholding and counting of wavelet coefficients. The feasibility of this framework is tested on a database of 2620 cheque images. This database consists of cheques from 10 different accounts. Each account is written by a different person.

\section{Customer Satisfaction Factor Extraction Method Using Text Mining}

Author: Yoko Kobayashi; Kazuhiko Tsuda

Specifically, the number of the part-time employees, who were atypical, the just-in- time employees, and the special employees increased. An atypical employment pattern is causing instability, given the case that payment and recognition are not commensurate with work and that the strong high production causes a negative image. It is disturbed by the atypical employment issues at most companies. Therefore, education by an enterprise is needed; this is ideally achieved in the short term through high efficiency.

\section{E. Free Text Mining of TCM Medical records Based On Conditional Random Fields}

Author: Qiyu Jiang; Hongyi Li; Jiafen Liang

Medical records of Traditional Chinese Medicine (TCM) are usually free text and unstructured data, how to extract medical terms from TCM medical records based on conditional random fields is an interesting problem.TCM medical records obtained from dermatology in Guangdong Provincial Hospital of Chinese Medicine are segmented to single words and labeled with grammatical properties of words by TCM expert, and divided into training sets and testing sets.

\section{Proposed System}

To identify emotion from the facial expression there are three steps they are: face detection, face extraction, classification of the emotions. Firstly we will detect the face by using viola Jones algorithm viola Jones algorithm is used for detecting the faces. Viola Jones is just giving a thousand no of faces to a computer or a digital camera that faces might be know faces or unknown faces to the computer once it get trained to identify whether it is human face or not then it will extract all the features and then classify.

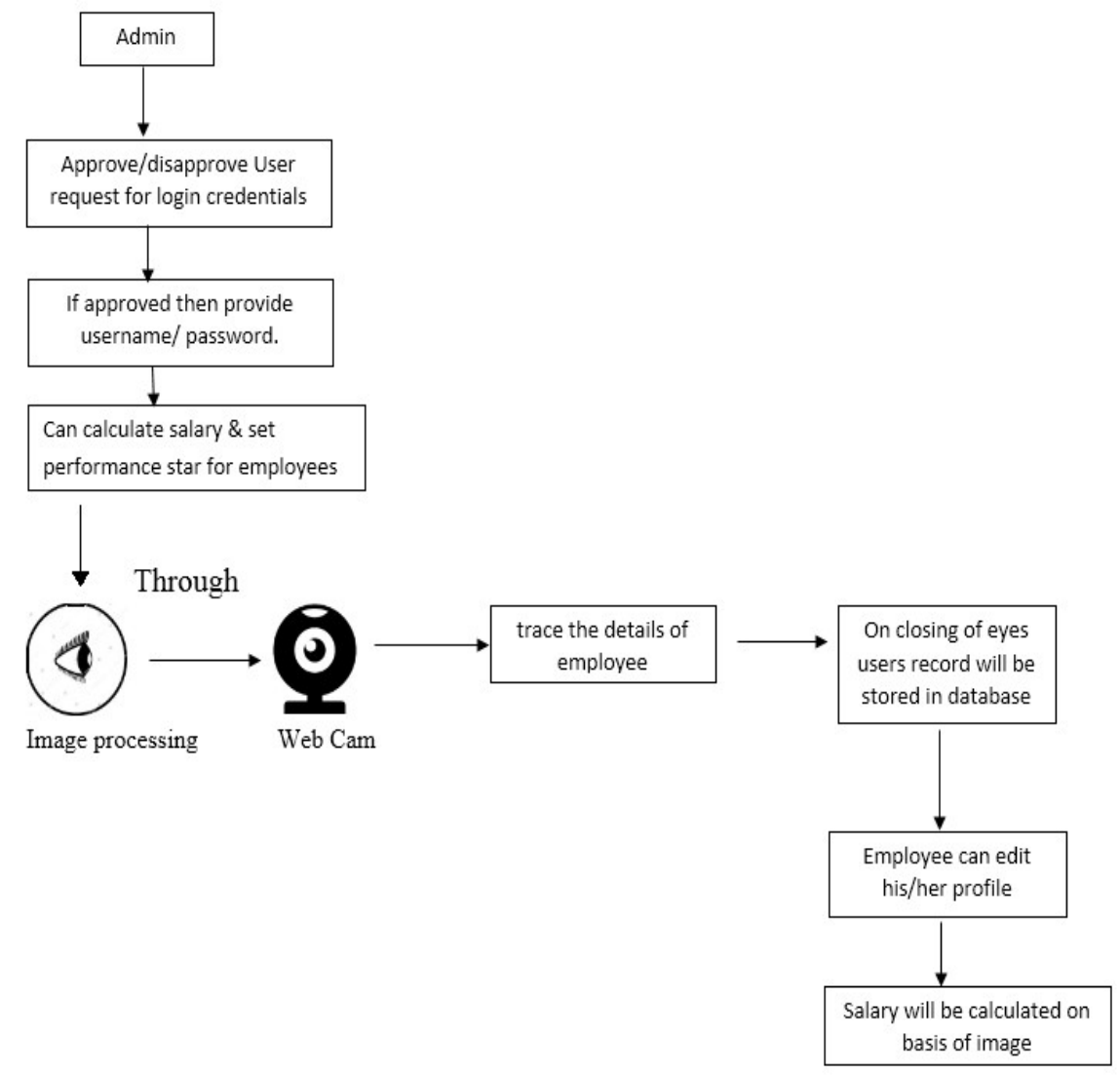


This system is used in maintaining employee data with the help of a database. Live Images from Web Camera and Text Messages will be sent while Employee is chatting with the colleague. With the help of image processing and face recognition algorithm we analyze mood of an employee. In this system we use NLP algorithm for sentiment of messages for emotion analysis. A Report of Employee based on Messages and Mood is generated automatically and shown to Admin of Employee Network. Our proposed approach is inspired from Hammal Calpier work and focuses on calculating six distances presented in Fig, between four parts of the face. Firstly, forty nine facial characteristic points in below fig. are detected using the SDM Method. Then just eleven characteristic points are considered to calculate six distances, and only the first and the last frames are considered from all sequence frames that represent respectively the neutral state and the emotion state. From these eleven detected points that are represented by $\mathrm{x}$ and $\mathrm{y}$ coordinates, and refer to the internal parts of the face, we have calculated Euclidian distances. After that, we have measured temporal deformation by calculating the ratio between frames; each calculated distance of the first frame is divided by the same calculated distance of the peak frame to calculate dynamic features.

\section{Methodology of the system:}

Face and Eye/Lip Detection Algorithm : This Algorithm detects Face from image frames. Eyes and Lips are detected using Image Processing from face.

Emotion Detection from Facial Expression Algorithm : This algorithm detects Emotion from Facial Expression by comparing current Eyes and Lip size with Normal Expression size of Eyes and Lip.

Emotion Detection Algorithm : This algorithm extracts keywords from text files containing different categories data and user messages are scanned to find whether they contain these keywords to classify messages into Happy/Angry/Sad/Confusing categories and also online API is used to get Emotion from Message so that Accuracy of Algorithm is increased.

NLP Algorithm : In this algorithm, four categories of sentiment are defined. User Message is parsed into Tree Structure and then words of message are analyzed from tree structure to find sentiment of message.

\section{Conclusion}

In this system with the help of image processing and face recognition algorithm we analyze mood of an employee. This system will use NLP algorithm for sentiment of messages for emotion analysis. A Report of Employee based on Messages and Mood will be generated. In image processing we use NLP algorithm. By this we will get to known mood of employee text processing the friend will chat with this another friend by that we will known the felling of the employee whether he is happy or not with his work.

\section{References}

1) Introduction to image processing http://www.engineersgarage.com..

2) Data mining-mining text datahttp://www.tutorialspoint.com/

3) Image retrieval using data mininghttp://www.ijireeice.com/

4) Sentiment analysis-https://en.wikipedia.org

5) Sentiment trend analysis in social web environment-http://www.ieeexplore.com

6) Real Time facial emotions recognition based on image processing and machine learninghttp://www.ijcaonline.org

7) Techniques and applications for sentiment analysis, communication of the ACM, 2013http://www.ieeeexplore.com

8) Ant colony optimization theory (ACO): a survey, theoretical computer science.

9) Facial expression and emotion databasehttp://www.mmk.ei.tum.html.

10) S. Srinivasan; H. Ranganathan; R. Srivel,'Employee monitoring \& HR management using RFID",2011 International Conference on Electronics, Communication and Computing Technologies, Year:

2011.

http://www.ieeeexplore.com

11) Priyankar Bhattacharjee"Work place monitoring of employee health using the workstation PC as a platform", 2014 IEEE Healthcare Innovation Conference (HIC),Year: 2014. http://www.ieeeexplore.com

12) Claas Richter; Yuzuru Tanaka; Gerald Bieber, "A case study of an employee portal with integrated physical-activity monitoring", 2011 4th International Conference on Human System Interactions, HSI 2011,Year: 2011. http://www.ieeeexplore.com 